\title{
Influencia del mentoring en el rendimiento de los participantes en las comunidades virtuales de práctica
}

\section{Influence of mentoring on the performance of the participants in online communities of practice}

\author{
Eneko Tejada Garitano \\ Universidad del País Vasco \\ eneko.tejada@ehu.eus \\ Urtza Garay Ruiz \\ Universidad del País Vasco \\ urtza.garay@ehu.eus \\ Ainara Romero Andonegui \\ Universidad del País Vasco \\ ainara.romero@ehu.eus
}

Recibido: 31/10/2017

Aceptado: $14 / 12 / 2017$

Publicado: 31/12/2017

\section{RESUMEN}

La Comunidad Virtual de Práctica (CVP) hace referencia a la red de personas que interactúan y comparten conocimientos con objeto de desarrollar su formación práctica y profesional. En este trabajo se presenta una investigación llevada a cabo con alumnado de Grado de Educación Primaria para conocer la influencia del mentoring en el rendimiento académico y en la satisfacción de participar en una CVP. Para ello, se desarrolló una CVP entre alumnado de diferente curso y cada estudiante construyó su Entorno Personal de Aprendizaje a través del aprendizaje colaborativo. La relación entre tutoría y rendimiento académico así como el nivel de influencia de la tutoría en el nivel de satisfacción del alumnado se analizó cuantitativamente. Entre los resultados destaca que los participantes valoran de forma satisfactoria la experiencia de participar e interactuar en una CVP, además de apreciar que la percepción que se tiene de la tutoría recibida no influye de forma directa en el rendimiento académico obtenido.

\section{PALABRAS CLAVE}

Comunidad Virtual de Práctica; mentoring; satisfacción; rendimiento académico.

\section{ABSTRACT}

Virtual Community of Practice refers to people who interact and share knowledge in order to develop their practical training. This paper describes the research carried out with Teaching Graduate students to measure the influence of mentoring on the academic performance and the satisfaction of participate in a VCP. To do this, students participated in a Virtual Community of Practice and each student created a Personal Learning Environment through peer tutoring and collaborative learning. Quantitative methodology was used to analyze the relationship between tutoring and academic performance and how tutoring has influence on student's satisfaction level. Results show that mentoring has not influence on academic performance and on the satisfaction with participating in a Virtual Community of Practice, but participants value satisfactorily the experience of taking part and interacting in a Virtual Community of Practice.

\section{KEYWORDS}

Virtual Community of Practice; mentoring; satisfaction; academic performance. 


\section{CITA RECOMENDADA}

Tejada, E., Garay, U. y Romero, A. (2017). Influencia del mentoring en el rendimiento de los participantes en las comunidades virtuales de práctica. RIITE. Revista Interuniversitaria de Investigación en Tecnología Educativa, 3, 40-50. Doi: http://dx.doi.org/10.6018/riite/2017/308571

Principales aportaciones del artículo y futuras líneas de investigación:

- Los resultados obtenidos a través de la investigación realizada permiten conocer la relación existente entre la tutoría y el rendimiento académico, y saber qué influencia tiene la tutoría en el nivel de satisfacción de los estudiantes que han participado en una Comunidad Virtual de Práctica.

- Estos hallazgos nos llevan a nuevas vías de estudio en las que nos gustaría profundizar:

- En líneas futuras, se espera analizar si la mejora en la orientación de los mentores mejora el grado de satisfacción del alumnado hacia su tutorización y ello correlaciona con el rendimiento académico.

\section{INTRODUCCIÓN}

A lo largo de los años las funciones y acciones que tanto profesorado como alumnado han llevado a cabo en el eLearning se han visto condicionadas por la evolución tecnológica de las plataformas sobre las que se ha apoyado (Conole, 2013; Downes, 2012). El desarrollo que han sufrido las formaciones online ha estado condicionado por las característicaS de los Content Management System (CMS), Learning Management Systems (LMS) y Learning Content Management Systems (CMLS) (Boneu, 2007; Gros 2011; Salinas, 2005) y también por la manera de entender Internet. Desde este último enfoque algunos autores como Castaño (2009), Llorente (2012) y Cabero (2013) constatan la existencia de una concepción pedagógica del eLearning desvinculada del concepto plataforma, y con una relación más estrecha con la forma de aprender a través de la tecnología que se basa en lo que se conoce como Web Social.

Desde esta perspectiva la web comparte el propósito que persiguen las herramientas sociales virtuales, lo que facilita que los usuarios de las aplicaciones Web 2.0 utilicen principalmente el software social en actividades de aprendizaje colaborativo para producir el suyo propio (Barroso y Cabero, 2013).

Los recursos que conforman el universo Web 2.0 tienden a ser dinámicos, simples, intuitivos y gratuitos (Moreno, 2012) y contribuyen a construir la inteligencia colectiva a través de una arquitectura que facilita la participación, siendo la interacción y el intercambio los pilares sobre los que se mantiene (Chen, Yen y Hwang, 2012). De esta forma, los usuarios, más allá de consumir contenidos, tienen la posibilidad de producir, editar y difundir información, compartir experiencias, materiales digitales o realizar actividades en un contexto virtual (Shin y Kim, 2008; Silva, 2011).

Fundamentalmente, lo que subyace bajo la filosofía de la Web Social es la forma o actitud de utilizar las herramientas de la Web 2.0, que conlleva implícitamente poner a disposición de otros usuarios los recursos generados y beneficiarse de sus producciones.

El desarrollo de herramientas que permiten trabajar de forma colaborativa en Internet ha contribuido a la proliferación de las Comunidades Virtuales (CV) (Geib, Braun, Kolbe y Brenner, 2004), es decir, a un tipo de redes sociales que tienen como fin aprovechar la inteligencia colectiva de sus componentes para generar nuevo conocimiento en la red (Pineda, Meneses y Téllez, 2013).

Una Comunidad Virtual de Aprendizaje hace referencia a un colectivo que comparte intereses comunes en la red, intercambia información, ideas, reflexiones y experiencias con objeto de desarrollarse de forma personal o académica y que contribuye a que otros lo hagan (Murua, Cacheiro y Gallego, 2015). Cuando esta comunidad se basa en la formación 
permanente (Wenger, McDermott, y Snyder, 2002) y el aprendizaje que se produce es en relación a la práctica en situaciones relacionadas con el trabajo, se considera Comunidad de Práctica (Desouza, Awazu y Baloh 2006; Moriones et al., 2013). Básicamente se trata de una forma de aprender socialmente apoyada en la participación colectiva que tiene como fin el desarrollo profesional de sus miembros (Pérez, 2015; Zhu y Baylen, 2005).

Las Comunidades de Práctica en contexto virtuales responden a la necesidad de desarrollar y diseñar ambientes online de aprendizaje colaborativo entre alumnado de diferente curso (Fernández-Sánchez y Valverde-Berrocoso, 2014; Schwier, Morrison y Daniel 2009). A través de ellas se fortalecen los conocimientos prácticos y el aprendizaje entre pares en la medida que los miembros de la comunidad con experiencia enseñan, aconsejan o guían a los demás. De esta forma los expertos se convierten en mentores de los participantes con menor bagaje (Cuenca-Amigo, García-Feijoo y Eizaguirre, 2016; Eby, 1997).

Para que en las Comunidades Virtuales de Práctica haya un alto grado de participación y éxito, los miembros que la configuran deben implicarse en la misma (Fernández-Sánchez y Valverde-Berrocoso, 2014). Para ello, es indispensable que ésta se establezca en relación a los intereses comunes de sus miembros, y que haya una comunicación eficaz que contribuya al conocimiento mutuo (Desouza, Awazu y Baloh 2006; Sallán y Del Pilar, 2006).

Así pues, el contexto virtual requiere de una química electrónica que permita crear cercanía y apego entre los participantes (Rísquez, 2010), que repetirán experiencia en la medida que se encuentren satisfechos de lo aprendido y perciban el aprendizaje realizado útil (Roca y Gagne, 2008).

La adquisición de conocimientos que se realiza a través de la interacción y tutorización de expertos resulta más atractiva que la que se basa en el autoaprendizaje clásico (Seoane y García-Peñalvo, 2014), por lo que la función de los mentores en las Comunidades Virtuales de Práctica orientadas a la formación del profesorado adquiere cada vez más importancia (Hou, 2015). Esta figura en entornos formales de formación como la universidad contribuye a la creación de un ecosistema de aprendizaje constructivista (Tang y Lam, 2014), ya que el mentor ayuda a los e-mentorizados a profundizar en sus fortalezas y debilidades, enfocando el proceso de relación en la experiencia que vive.

El resultado de esta relación es beneficioso tanto para el mentor como para el mentorizado. Para el primero porque mejora su metacognición (Galbraith y Winterbottom, 2011) y auto-regulación (De Backer, Van Keer y Valcke, 2012), desarrolla habilidades sociales y de orientación (Moraga 2011; Reddick, Griffin, Cherwitz, Cérda-Pražák y Bunch, 2012) e incrementa su autoestima y satisfacción personal (Valverde, Ruiz, García y Romero, 2004). Para el segundo porque gracias a la motivación que obtiene a través de este tipo de ayuda mejora su rendimiento académico (Kregor, Breslin y Fountain, 2012).

En cualquiera de los casos, las experiencias educativas generadas en las Comunidades de Práctica repercuten de forma positiva tanto en los resultados académicos de los mentorandos como de los mentores (Ginsburg-Block, Rohrbeck y Fantuzzo, 2006; Soto, Cuadrado, García, Rísquez, y Ortega, 2012; Tremblay y Rodger, 2003).

En este artículo se presenta una investigación realizada en la Universidad del País Vasco (UPV/EHU), cuyo objetivo es conocer la influencia del mentoring en el rendimiento académico y en la satisfacción del alumnado participante de una Comunidad Virtual de Práctica. Para este fin se plantearon las siguientes preguntas de investigación:

1. ¿Existe relación entre la tutoría y el rendimiento académico?

2. ¿Influye la tutoría en el nivel de satisfacción de los estudiantes que han participado en la Comunidad Virtual de Práctica? 


\section{MÉTODO}

\subsection{Muestra}

La muestra no probabilística de corte incidental del análisis que se presenta a continuación la conformaron 147 estudiantes de la asignatura de Función Docente del primer curso del Grado de Educación Primaria de la Escuela de Magisterio de Bilbao (UPV/EHU).

Para evaluar la influencia del mentoring en el rendimiento académico y en la satisfacción del alumnado participante de una Comunidad Virtual de Práctica y con objeto de responder a las preguntas de investigación, se llevó a cabo un estudio de carácter cuantitativo descriptivo y correlacional.

\subsection{Instrumentos}

Los datos se recogieron a través del cuestionario Technology Acceptance Model (TAM) (Wojciechowski y Wojciech, 2013) adaptado a las características de la experiencia educativa desarrollada. Se puso un interés especial en las variables que componen este instrumento. La satisfacción en relación a la experiencia educativa en un entorno virtual de práctica basada en el mentoring y la valoración de la tutoría recibida fueron medidas por medio de 7 ítems a través de una escala likert ( 1 =totalmente desacuerdo y $10=$ totalmente acuerdo).

Primera dimensión: Valoración de la tutoría recibida

El tutor ha realizado aportaciones y sugerencias.

El tutor ha tenido una aptitud y actitud positiva.

El tutor ha motivado.

El tutor ha orientado adecuadamente.

El tutor ha mostrado ser experto en la materia.

El tutor ha favorecido la reflexión individual y/o grupal. virtual.

Segunda dimensión: Satisfacción en relación con la experiencia educativa en un entorno

La participación en esta experiencia educativa ha sido positiva.

Para realizar el análisis se utilizó el programa estadístico SPSS versión 22.

\subsection{Procedimiento}

La investigación se desarrolló en la Escuela Universitaria de Magisterio de Bilbao, concretamente en dos asignaturas del Grado de Educación Primaria: la primera denominada Función Docente, que se imparte en el primer curso, y la segunda, Lenguas e innovación en la escuela, que forma parte del Minor de especialización titulado La Diversidad Lingüística en la Escuela y que se imparte en cuarto curso.

El trabajo presentado es de corte cuantitativo, basado en un análisis descriptivo y correlacional para medir la relación entre las variables: tutoría recibida, rendimiento académico y satisfacción en relación a la experiencia de aprendizaje en una comunidad online de prácticas.

La innovación educativa consistía en que el alumnado construyese un entorno personal de aprendizaje (PLE) mediante la inserción y desarrollo de una Comunidad Virtual de Práctica (CVP) cuyo objetivo final era que el alumnado participante reconociese cómo, con quién y con qué aprende. Es decir, crear un contexto educativo donde cada estudiante se hiciera consciente de su propio proceso de aprendizaje.

Para lograr el objetivo señalado, se diseñaron y desarrollaron 3 fases:

\section{FASE 1. Crear una Comunidad Virtual de Práctica}

Se creó una Comunidad Virtual de Práctica en la que participó tanto el profesorado como el alumnado de primero y cuarto curso. En el desarrollo de la Comunidad se puso en contacto a 47 estudiantes de cuarto curso con 147 de primero mediante una red social. A través de la 
Comunidad Virtual de Práctica se impulsó la realización de proyectos que tenían como fin dar a conocer entre los participantes de la red los trabajos realizados para la asignatura señalada, lo que facilitó la cooperación entre estudiantes y contribuyó a la autonomía personal de cada participante en su proceso de aprendizaje. Todas estas acciones se sustentaron en el dominio, la comunidad y la práctica (Castañeda y Adell, 2011).

Para la realización de estas actividades se eligió la red social NING que poseía características adecuadas para este tipo de actividad, fundamentalmente privacidad y multiplicidad de herramientas comunicativas. A través de ella, los participantes compartieron preocupaciones, pasiones y curiosidades, aprendiendo y profundizando en los conceptos de la asignatura Función Docente mientras interactuaban regularmente online.

Por otra parte, la misma red social, además de las que el alumnado ya utilizaba en su vida cotidiana, fue utilizada por el conjunto de alumnos y alumnas para comunicarse virtualmente tanto de forma síncrona y asíncrona, en privado y en público.

\section{FASE 2. Asignar los roles y funciones de los mentores y mentorizados}

Los profesores de las asignaturas que participaron en el PIE otorgaron el rol de tutor al alumnado de cuarto curso para que realizase las funciones propias de un mentor y guiase a los estudiantes de primer curso en la elaboración de producciones basadas en el desarrollo de los contenidos de la asignatura Función Docente, de la que habían sido alumnos hacía cuatro años.

De esta forma, cada estudiante de cuarto realizó mentoring a un grupo de 4 o 5 estudiantes de primero en la creación de sus proyectos de aprendizaje respecto a los siguientes cuatro centros de interés de la asignatura "Función Docente":

Identidad personal y profesional del profesor.

Funciones y labores del profesorado.

Razones para elegir ejercer de profesor.

Recorrido profesional del profesor.

Así, dependiendo del tipo de proyecto, los tutores orientaron al alumnado de primer curso en la elección de las herramientas más apropiadas de la Web 2.0, aportando sugerencias y fomentando la reflexión y la motivación.

FASE 3. Compartir lo aprendido y coevaluarlo en la Comunidad

Una vez realizada la producción o proyecto, ésta fue puesta a disposición de la comunidad educativa (194 estudiantes), en la red social Ning, con el fin de que fuese valorada y recibir el feedback por parte de los miembros de la comunidad. A través de la evaluación entre alumnado de diferente curso se pretendía fomentar la interacción y la creación de significados comunes, incrementando así la intencionalidad formativa de la colaboración y cooperación entre los estudiantes en la Comunidad.

\section{RESULTADOS}

Tras la realización del análisis estadístico descriptivo y correlacional, los resultados se describen en torno a las dos preguntas de investigación planteadas.

A través de la primera pregunta de investigación (1. ¿Existe relación entre la tutoría y el rendimiento académico?) se pretende conocer la relación existente entre la valoración global de la tutoría recibida y el rendimiento académico obtenido.

Del estudio de los resultados se extrae que cerca de la mitad del alumnado $(46,9 \%)$ se encuentra altamente satisfecha $(\geq 8 \leq 10)$ con la tutoría recibida $(M 8,6)$. No obstante, los datos también muestran que el $29,3 \%$ de los estudiantes ha valorado $(M 6,619)$ esta cuestión dentro del rango medio $(>5<8)$ y un $23,7 \%(\mathrm{M} 3,353)$ de forma baja $(<5)$.

Respecto al rendimiento académico se extrae que la puntuación media que ha obtenido el alumnado tutorizado del primer curso es de 6,89 y que casi la mitad de estos estudiantes $(45,5 \%)$ ha logrado una puntuación superior $(M 7,248)$. También se evidencia que el porcentaje 
de alumnos y alumnas que ha obtenido un resultado $(M \quad 8,498)$ dentro del rango alto de puntuaciones es del $30,7 \%$, y casi otra cuarta parte $(23,8 \%)$ ha rendido académicamente por debajo del cinco (M 4,147).

Una vez realizado el estudio correlacional (coeficiente de Pearson) entre la valoración global de la tutoría recibida y el rendimiento académico, se observa que la relación lineal entre ambas variables es leve ( $R$ de Pearson 0,059$)$ por lo que no hay influencia directa entre ambas variables.

En relación a la segunda pregunta de investigación (2. ¿Influye la tutoría en el nivel de satisfacción de los estudiantes que han participado en la Comunidad Virtual de Práctica?), los datos muestran que la $R$ de Pearson es de 0,291 (Correlación significativa en el nivel 0,01), por lo que no hay una relación lineal entre la tutoría global recibida y la satisfacción de los estudiantes.

Los resultados también ponen de manifiesto que el alumnado se encuentra satisfecho respecto a la tarea realizada y el haber participado en la Comunidad Virtual de Prácticas (M 7,9 ; DT 1,02 ) ya que el $72 \%$ lo ha valorado en el rango alto y sólo el $2,1 \%$ en el rango bajo.

Respecto a la tutoría recibida por parte del alumnado de primero, el aspecto más valorado es el que se refiere a la realización de aportaciones y sugerencias que ha realizado el tutor ( $\mathrm{M}$ $7,287)$ que además el $58,8 \%$ ha valorado dentro del rango alto $(\geq 8 \leq 10)$. En relación a esta cuestión en orden descendente de valoración, se encuentra la aptitud y actitud del tutor hacia el alumnado mentorizado $(50,9 \%)$, la motivación que ha recibido del tutor $(49 \%)$, la orientación que ha llevado a cabo éste $(47,7 \%)$, y por último el hecho de que el tutor haya favorecido la reflexión individual y/o grupal.

También se observa que el grado de dispersión que ha tenido el alumnado a la hora de valorar los diferentes aspectos de la tutoría recibida es cercano a los 2,5 puntos (ver Tabla 1). Sin embargo, como el coeficiente de variación (desviación típica / media) de las diferentes medias no es superior a 1 y se encuentra más cercano al 0 , hay poca dispersión entre los valores de la variable y por lo tanto la media de la muestra es suficientemente representativa.

Tabla 1. Medidas de valoración del alumnado al tutor experto

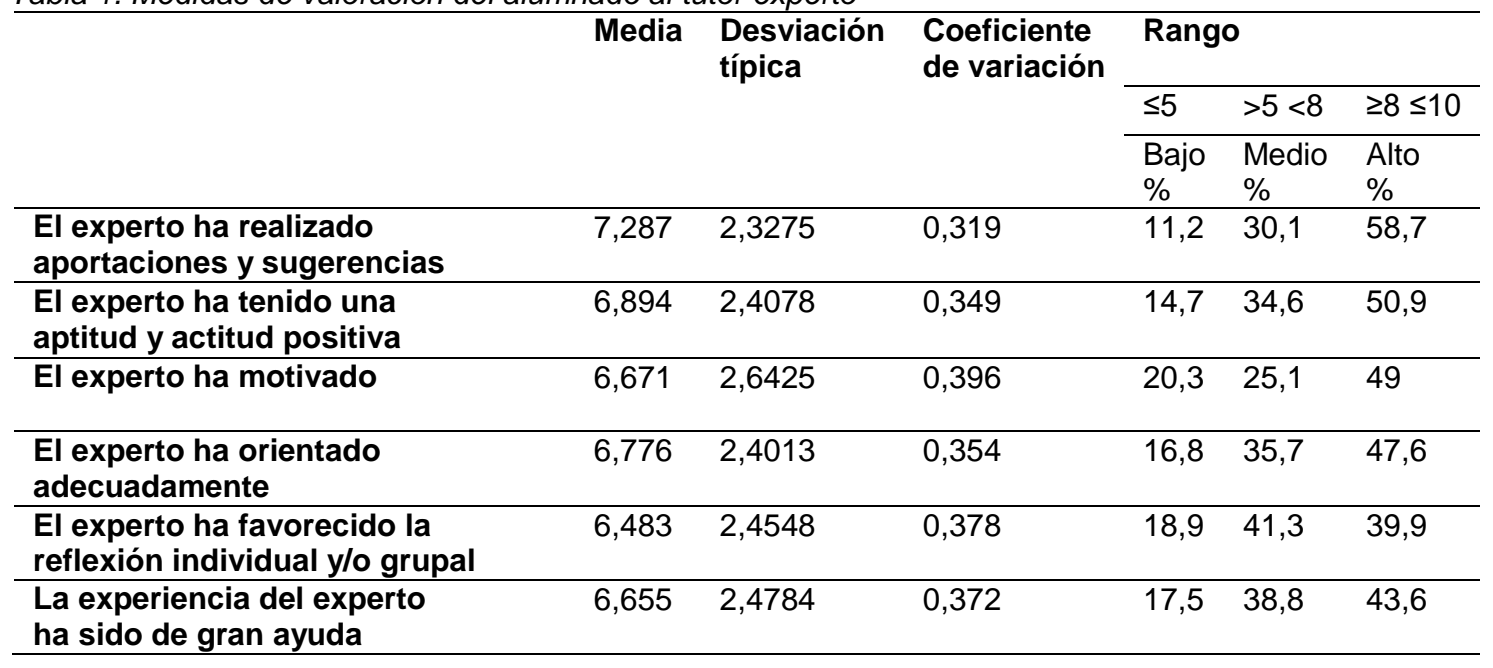

Por tanto, el hecho de que el alumnado valore de forma positiva la tarea realizada y el haber participado en la Comunidad Virtual de Práctica es independiente a la satisfacción que muestran respecto al papel que han desarrollado sus tutores.

\section{DISCUSIÓN Y CONCLUSIONES}

Las Comunidades Virtuales de Práctica permiten diseñar ambientes online de aprendizaje colaborativo entre alumnado de diferente curso (Fernández-Sánchez y Valverde-Berrocoso, 2014; Schwier, Morrison y Daniel 2009), es decir desarrollar prácticas de mentoring entre 
alumnos que tienen diferente nivel de conocimiento. Este tipo de tutorización ayuda al estudiante que recibe los consejos y orientaciones por parte de su mentor con el objetivo final de mejorar su rendimiento académico (Soto, Cuadrado, García, Rísquez, y Ortega. 2012; Tremblay y Rodger, 2003).

En la investigación realizada se constata en que casi la mitad del alumnado que ha participado en la Comunidad Virtual de Prácticas ha obtenido un rendimiento académico notable y se encuentra altamente satisfecho con la tutoría recibida, a pesar de que no se verifica la relación existente entre rendimiento académico, valoración y tutoría recibida. Esto es, participar en este tipo de entornos de aprendizaje parece promover la mejora del rendimiento. Aspecto que no está ligado a la valoración o percepción que presenta el alumnado con la tutoría recibida.

De forma pormenorizada, se infiere que los estudiantes del primer curso valoran del conjunto de acciones que realiza el tutor, sobre todo, las aportaciones y sugerencias que realiza para trabajar mejor, así como su aptitud y actitud positiva. Sin embargo, tal y como se ha señalado, los datos del estudio llevado a cabo ponen de manifiesto que no existe una relación lineal o influencia directa entre ambas variables, por lo que no se puede concluir que en una Comunidad Virtual de Prácticas la valoración de la tutoría determine o condicione el rendimiento académico. Aspecto que nos dirige a seguir investigando en lo que ocurre en este tipo de entornos y en el análisis de cuáles pueden ser los factores que influyen en el rendimiento académico del alumnado participante.

En este línea, cabe la posibilidad que aspectos como el nivel de competencia tecnológica y comunicativa online (Cabero y Llorente, 2008; Cabero y Romero, 2010), o el nivel de aprovechamiento y utilización de los recursos de la Web 2.0 (Cabero, 2006), haya tenido mayor peso en el rendimiento académico del alumnado que el mentoring.

A través de este estudio también se ha podido constatar que no existe una relación lineal entre la tutoría global recibida y la satisfacción de los estudiantes en relación a la experiencia que han tenido de trabajar en una Comunidad Virtual de Prácticas. Es decir, el alumnado ha juzgado la experiencia educativa independientemente de la percepción de la calidad de la tutoría recibida por los estudiantes de cuarto curso. No en vano, casi tres de cada cuatro estudiantes se encuentran satisfechos de la tarea realizada y de haber participado en la Comunidad Virtual de Prácticas.

Lo que nos lleva a pensar que la experiencia de haber participado en una CVP ha respondido a los intereses comunes de sus miembros (Sallán y Del Pilar, 2006), a la utilidad percibida del aprendizaje (Arbaugh, 2002; Roca, Chiu y Martínez, 2006; Roca y Gagne, 2008), a la interacción comunicativa a través de herramientas virtuales (Cebrian de la Serna, 2011), y a la tecnología y diseño de la formación (Gallagher y Newman, 2002; Hannon y D’Netto, 2007; Sun, Tsai, Finger, Chen, y Yeh, 2008), y la tutoría recibida no se constituye como elemento básico en estos procesos de aprendizaje. Por tanto, el uso de Comunidades Virtuales de Práctica en la enseñanza universitaria es una herramienta positiva para el alumnado universitario, independientemente del rendimiento académico del alumnado participante y de la valoración de tutoría recibida.

Finalmente, tras la reflexión e investigación realizadas, sería interesante evaluar el aprendizaje y la satisfacción del alumnado tutor también, ya que, su participación en Comunidades de Aprendizaje Virtuales en calidad de expertos mejora su metacognición y autoregulación (De Backer, Van Keer y Valcke, 2012; Galbraith y Winterbottom, 2011).

\section{RECONOCIMIENTOS}

Proyecto de innovación educativa (PIE) denominado "EHUPLE2015: Creación de Entornos Personales de Aprendizaje (PLE), desde la cooperación y colaboración en Comunidades Virtuales de Práctica formadas por alumnado y profesorado del Grado de Educación Primaria" (6722), financiado por la UPV/EHU. 


\section{REFERENCIAS BIBLIOGRÁFICAS}

Arbaugh, J. B. (2002). Managing the on-line classroom: a study of technological and behavioral characteristics of web-based MBA courses. Journal of High Technology Management Research, 13, pp. 203-223. doi: 10.1016/S1047-8310(02)00049-4

Barroso, J. y Cabero, J. (2013). Replanteando el e-learning: hacia el e-learning 2.0. Campus Virtuales, 2 (2), pp. 76-87.

Boneu, J. M. (2007). Plataformas abiertas de e-learning para el soporte de contenidos educativos abiertos. RUSC. Universities and Knowledge Society Journal, 4(1). Recuperado de http://www.raco.cat/index.php/RUSC/article/view/58133/68225

Cabero, J. (2006). Comunidades virtuales para el aprendizaje. Su utilización en la enseñanza. Edutec, $\quad X X$. Recuperado de http://www.edutec.es/revista/index.php/edutece/article/viewFile/510/244

Cabero, J. y Llorente, M.C. (2008). La alfabetización digital de los alumnos. Competencias digitales para el siglo XXI. Revista Portuguesa de pedagogía. 42(2), pp. 7-28. Recuperado de http://tecnologiaedu.us.es/cuestionario/bibliovir/jca26.pdf

Cabero, J. y Romero, R. (2010). Análisis de buenas prácticas del e-learning en las universidades andaluzas. Revista Electrónica Teoría de la Educación: Educación y Cultura en la Sociedad de la Información, 1(1), pp. 283-309. Recuperado de http://www.redalyc.org/articulo.oa?id=201014897012

Cabero, J. (2013). La formación virtual en el nuevo entramado 2.0: el e-learning 2.0. En I. Aguaded, y J. Cabero, Tecnologías y medios para la educación en la e-sociedad (pp. 2352). Madrid: Alianza Editorial.

Castañeda, L. y Adell, J. (2011). El desarrollo profesional de los docentes en entornos personales de aprendizaje (PLE). En Roig Vila, R. y Laneve, C. (Eds.), La práctica educativa en la Sociedad de la Información: Innovación a través de la investigación (pp. 83-95). Alcoy: Marfil.

Castaño, C. (2009). Retos para el aprendizaje y la investigación en el elearning 2.0. En C. Castaño (Cood.), Web 2.0. El uso de la Web en la sociedad del conocimiento, (pp. 31-55). Caracas: Universidad Metropolitana.

Cebrian de la Serna, M. (2011). Supervisión con e-portafolios y su impacto en las reflexiones de los estudiantes en el Practicum. Estudio de caso. Revista de Educación, 354, pp. 183208.

Chen, S. C., Yen, D. C. y Hwang, M. I. (2012). Factors influencing the continuance intention to the usage of Web 2.0: An empirical study. Computers in Human Behavior, 28(3), pp. 933941.

Conole, G. (2013). Digital identity and presence in the social milieu. Paper presented at the Pelicon conference, Plymouth.

Cuenca-Amigo, M., García-Feijoo, M. y Eizaguirre, A. (2016). Reflexión sobre el mentoring como experiencia de ocio educativo y social en personas mayores de 65 años. Revista Interuniversitaria de Formación del Profesorado, 30(2), pp. 77-88.

De Backer, L., Van Keer, H. y Valcke, M. (2012). Exploring the potential impact of reciprocal peer tutoring on higher education students' metacognitive knowledge and regulation. Instructional science, 40 (3), pp. 559-588.

Desouza, K. C., Awazu, Y. y Baloh, P. (2006). Managing knowledge in global software development efforts: Issues and practices. IEEE software, 23 (5), pp. 1-30. 
Downes, S. (2012). E-Learning generations. Recuperado de http://halfanhour.blogspot.be/2012/02/elearning-generations.html

Eby. L.T. (1997). Alternative forms of mentoring in changing organizational environments: A conceptual extension of the mentoring literature. Journal of Vocational Behavior, 51, pp. 125-144. doi: 10.1006/jvbe.1997.1594

Fernández-Sánchez, M. R. y Valverde-Berrocoso, J. (2014). Comunidades de práctica: un modelo de intervención desde el aprendizaje colaborativo en entornos virtuales. Comunicar: Revista Científica de Comunicación y Educación, 21(42), 97-105.

Galbraith, J. y Winterbottom, M. (2011). Peer-tutoring: what's in it for the tutor? Educational Studies, 37(3), pp. 321-332.

Gallagher, S. y Newman, A. (2002). Distance learning at the tipping point. Critical success factors to growing fully online distance learning programs. Boston: Eduventures.

Geib, M., Braun, C., Kolbe, L. y Brenner, W., (2004). Measuring the Utilization of Collaboration Technology for Knowledge Development and Exchange in Virtual Communities. In System Sciences, 2004. Proceedings of the 37th Annual Hawaii International Conference, pp. 1-10.

Ginsburg-Block, M. D., Rohrbeck, C. A. y Fantuzzo, J. W. (2006). A meta-analytic review of social, self-concept, and behavioral outcomes of peer-assisted learning. Journal of Educational Psychology, 98(4), pp. 732.

Gros, B. (2011). Evolución y reto de la educación virtual. Construyendo el elearning del siglo XXI. Barcelona: UOC. Recuperado de http://openaccess.uoc.edu/webapps/o2/bitstream/10609/9781/1/TRIPA elearning castellano.pdf

Hannon, J. y D'Netto, B. (2007). Cultural diversity online: student engagement with learning technologies. International Journal of Educational Management, 21(5), 418-432.

Hou, H. (2015). What makes an online community of practice work? A situated study of Chinese student teachers' perceptions of online professional learning. Teaching and Teacher Education, 46, pp. 6-16. doi: 10.1016/j.tate.2014.10.005

Kregor, G., Breslin, M. y Fountain, W. (2012). Experience and beliefs of technology users at an Australian university: Keys to maximizing e-learning potential. Australasian Journal of Educational Technology, 28(8), pp. 1382-1404.

Llorente, C. (2012). El elearning 2.0: de la tecnología a la metodología. @tic. revista d'innovació educativa, 9, pp. 79-86. doi: 10.7203/attic.9.1939

Moraga, D. (2011). Mentoring: Estrategia de liderazgo para el policía del siglo XXI. Revista Estudios Policiales, 8, pp. 99-107.

Moreno, A. J. (2012) Web Social-Recurso Educativo. Observatorio Tecnológico. Ministerio de Educación Cultura y Deporte. Madrid, España. Recuperado de http://recursostic.educacion.es/observatorio/web/es/internet/web-20/1060-la-web-20recursos-educativos.

Moriones, E. R., Golinelli, G. F., López, M. G., Fernández, E. G., Esteban, A. B., Sánchez, M. A. y De Benito, M. V. (2013). Evolución de una comunidad de prácticas virtual para la mejora de la docencia online. Actas de las $X$ Jornadas Internacionales de Innovación Universitaria (JIIU 2013).

Murua, I., Gallego, D. y Cacheiro, M. L. (2015). Caracterización de las cibercomunidades de aprendizaje (cCA). Revista de Educación a Distancia, 47, pp. 1-18. doi: 10.6018/red/47/4 
Pérez, M. A. G. (2015). Enriquecimiento tecnológico y psicopedagógico del concepto de comunidades de práctica en la educación a distancia. Revista de Educación a Distancia, 47. Recuperado de http://revistas.um.es/red/article/view/242421/184241

Pineda, E., Meneses, T. y Téllez, F. R. (2013). Análisis de redes sociales y comunidades virtuales de aprendizaje. Antecedentes y perspectivas. Revista Virtual Universidad Católica del Norte, 1(38), 40-55.

Reddick, R., Griffin, K., Cherwitz, R., Cérda-Pražák, A. y Bunch, N. (2012). What you get when you give: how graduate students benefit from serving as mentors. The Journal of Faculty Development, 26(1), pp. 37-49.

Rísquez, A. (2010). La mentoría electronica entre pares para la adaptación a la universidad: Una experiencia de investigación aplicada (Tesis doctoral). Universidad Nacional de Educación a Distancia, Madrid.

Roca, J. C. y Gagné, M. (2008). Understanding e-learning continuance intention in the workplace: A self-determination theory perspective. Computers in Human Behavior, 24(4), pp. 1585-1604. doi: 10.1016/j.chb.2007.06.001

Roca, J., Chiu, C. y Martínez, F. (2006). Understanding e-learning continuance intention: An extension of the Technology Acceptance Model. International Journal of human-computer studies, 64(8), pp. 683-696. doi: 10.1016/j.ijhcs.2006.01.003

Salinas, J. (2005). La gestión de los entornos virtuales de formación. En Seminario Internacional sobre La Calidad de la Formación en Red en el Espacio Europeo de Educación Superior. Tarragona, España. de http://gte.uib.es/pape/gte/sites/gte.uib.es.pape.gte/files/gestioEVEA 0.pdf

Sallán, J. G., y del Pilar, M. (2006). Análisis de la interacción en comunidades virtuales. Educar, 37, pp. 125-150.

Schwier, R.A., Morrison, D. y Daniel, B.K. (2009). A preliminary investigation of self-directed learning activities in a non-formal blended learning environment. Paper presented at the American Educational Research Association, San Diego, CA. Recuperado de http://es.scribd.com/doc/14224752/A-Preliminarylnvestigation-of-Self-Directed-LearningActivities-in-a-Non-Formal-BlendedLearning-Environment

Seoane, A. M. y García-Peñalvo, F. J. (2014). Patrones pedagógicos y docencia en red. En J. A. Jerónimo Montes (Ed.), Formación en red: aprender con tecnologías digitales (pp. 3047). México: Universidad Nacional Autónoma de México.

Shin, D.H. y Kim, W.Y. (2008). Applying the technology acceptance model and flow theory to cyworld user behavior: Implication of the Web2.0 user acceptance. Cyberpsychol. Behav. 11 , pp. 378-382.

Silva, Q. (2011). Diseño y moderación de entornos virtuales de aprendizaje (EVA). Barcelona: UOC.

Soto, N. M., Cuadrado, A. M., García, M. S., Rísquez, A. y Ortega, M. S. (2012). El rol del mentor en un proceso de mentoría universitaria. Educación XX1, 1 (2), pp. 93-118.

Sun, P. C., Tsai, R. J., Finger, G., Chen, Y. Y. y Yeh, D. (2008). What drives a successful eLearning? An empirical investigation of the critical factors influencing learner satisfaction. Computers \& education, 50(4), pp. 1183-1202.

Tang, E y Lam, C. (2014). Building an effective online learning community (OLC). In blogbased teaching portfolios. The Internet and Higher Education, 20, pp. 79-85. doi: 10.1016/j.iheduc.2012.12.002 
Tremblay, P. F. y Rodger, S. (2003). The effects of a peer mentoring program on academic success among first year university students. The Canadian Journal of Higher Education, 33(3), pp. 1-17.

Valverde, A., Ruiz de Miguel, C., García Jiménez, E. y Romero, S. (2004). Innovación en la orientación universitaria: La mentoría como respuesta. Contextos Educativos, 6-7, pp. 87112.

Wenger, E., McDermott, R. y Snyder, W. M. (2002). Cultivating Communities of Practice: A guide to Managing Knowledge. Boston: Harvard Business School Press.

Wojciechowski, R. y Wojciech, C. (2013). Evaluation of learners'attitude toward learning in ARIES augmented reality environments. Computers \& Education, 68, pp. 570-585. doi: 10.1016/j.compedu.2013.02.014

Zhu, E. y Baylen, D. M. (2005). From learning community to community learning: pedagogy, technology and interactivity. Educational Media International, 42(3), pp. 251-268.

\section{INFORMACIÓN SOBRE LOS AUTORES}

\section{Eneko Tejada Garitano \\ Universidad del País Vasco}

Eneko Tejada Garitano, es doctor por la Universidad del País Vasco y profesor de la Escuela Universitaria de Magisterio de Bilbao y del Máster Universitario Internacional Aprendizaje, Tecnología y Educación de la UPV/EHU. Forma parte del grupo de investigación consolidado Weblearner, y ha participado en diferentes proyectos de $i+d+i$ nacionales e internacionales. Ha desempeñado de forma ininterrumpida una actividad investigadora en el área de la educación, centrada en la aplicación de las nuevas tecnologías a la educación. También ha publicado varios artículos en revistas nacionales e internacionales de diferente indexación. Fuera del ámbito universitario, imparte cursos de formación a profesorado en activo de Educación Infantil, Primaria y Secundaria sobre, metodologías activas y nuevas tecnologías.

\section{Urtza Garay Ruiz \\ Universidad del País Vasco}

Urtza Garay Ruiz es Directora de Innovación Pedagógica de la Universidad del País Vasco. Es doctora y profesora de la Escuela Universitaria de Magisterio de Bilbao. Es profesora en Grados de Educación, en el Máster Universitario Aprendizaje, Tecnología y Educación y Máster de Enseñanza Secundaria, Bachillerato y Enseñanza de Idiomas de la UPV/EHU. Sus líneas de investigación están centradas en la Tecnología Educativa, Formación Universitaria, MOOC, PLE, Realidad Aumentada, Web 2.0 y Movimiento Maker en educación. Ha publicado varios artículos en revistas indexadas como JCR, además de más de 20 artículos en revistas nacionales e internacionales de diferente indexación (SCOPUS, ISI, etc.). Participa habitualmente como comunicante y ponente invitada en Congresos Internacionales de renombre. Es autora de material didáctico para niveles educativos desde Educación Primaria, Secundaria y Universidad).

\section{Ainara Romero Andonegui \\ Universidad del País Vasco}

Ainara Romero Andonegui, Doctora en Educación por la Universidad del País Vasco y Profesora en la Escuela de Magisterio de Bilbao de la UPV/EHU. Su área de conocimiento e investigación se centra en la Tecnología Educativa y la Adquisición del lenguaje. Forma parte del grupo de investigación consolidado Weblearner, y ha participado en diferentes proyectos de $i+d+i$ nacionales e internacionales. En cuanto a la actividad profesional, ha impartido docencia tanto en las etapas de Infantil y Primaria, así como en la Universitaria. Se subraya el papel como docente en contextos de formación online (MiriadaX, OCW).

\section{(cc) EY-NO}

Los textos publicados en esta revista están sujetos a una licencia de Reconocimiento 4.0 España de Creative Commons. Puede copiarlos, distribuirlos, comunicarlos públicamente y hacer obras derivadas siempre que reconozca los créditos de las obras (autoría, nombre de la revista, institución editora) de la manera especificada por los autores o por la revista. La licencia completa se puede consultar en:Licencia Creative Commons Atribución-NoComercial-Compartir por igual 4.0 Internacional. 